\title{
Thermal diffusivity estimation with averaged infrared thermography
}

\author{
by E. Poncet ${ }^{1}$, D. Béreiziat ${ }^{1}$, G. Grangeot ${ }^{1}$ and J.-C. Batsale ${ }^{2}$ \\ 'U.F.C. - I.G.E., Parc Technologique - 2, Av. Jean Moulin, 90000 Belfort, France \\ ${ }^{2}$ L.E.P.T.- E.N.S.A.M. (U.R.A. C.N.R.S. 873), Esplanade des Arts et Métiers, 33405 Talence Cedex, \\ France
}

\begin{abstract}
Evaluation methods are proposed for thermal characterisation of layered systems by means of quantitative infrared measurements. Two thermal configurations are studied with an averaging procedure for two layer system. The principle of the estimation method is based on the use of Taylor series of the non-linear analytical transformation solutions to apply linear estimators to fit spectral distributions or time evolutions to experimental macroscopic temperature data. The advantage for potential industrial and laboratory applications is related to non-destructive thermal evaluation of delaminations in composite laminate and to thermophysical characterisation, i.e., diffusivity identification.
\end{abstract}

\section{Introduction}

Unlike contact sensor systems, infrared temperature measurements can provide useful data for an accurate quantitative characterisation of plane systems. However the measurement noise of the IRT camera has to be reduced strongly [1, 2]. Averaging processes allow for such a noise reduction by averaging temperature fields that are computed from a sequence of infrared temperature fields.

In this paper, two methods are proposed for the thermal characterisation of layered systems from averaged temperature data. The configurations of the thermal problems, i.e., steady or transient heat conduction with uniform heat losses, are described by analytical solutions obtained with integral transform methods in the Fourier or Laplace spectral domains. These solutions are linearised on the basis of a Taylor series to provide approximate models that are well suited to methods of linear parameter estimation. The analytical identification function obtained this way involves the thermal properties of both phases and a potential thermal contact resistance, which is directly linked to the averaged temperatures of the phases. Therefore averaged temperature data can be used to investigate these parameters. Averaging processes of temperature fields measured with an infrared camera are appropriate to these averaging descriptions of the heat transfer. The aim of these new techniques is to propose analytical and direct methods for characterisation of layered systems at room temperature. The main advantage of this method is to process a large number of data in order to reduce the effect of the measurement noise on the estimation result. Fields of application of the non-destructive thermal evaluation of delaminations in composite laminate and thermophysical characterisation are covered by these methods. Indeed, thermal properties of homogeneous media or thermal contact resistance in layered composites can be determined with a simple experimental fit sample, since neither the knowledge of the heat flux excitation nor a sophisticated insulating apparatus is required.

\section{Thermal problem}

The heat diffusion problem for a layered medium with the geometrical features is presented in Fig. 1. Two adjacent stagnant phases are considered with a perfect conducting contact or a uniform thermal contact resistance $R_{c}$. The parallelepiped system is submitted to a continuous or transient local heat source Q1 on the 1-phase and to uniform heat losses 
(xy-planes) characterised by a convective heat coefficient $h_{c}$, to verify the fin hypothesis. The steady and transient three-dimensional analytical problems are solved and the corresponding analytical transformed solutions are developed. We consider both layers made of homogeneous, isotropic solids with temperature-independent properties. The thermal problem is described by the following differential equation system:

$$
\frac{\partial^{2} T_{i}}{\partial x^{2}}+\frac{\partial^{2} T_{i}}{\partial y^{2}}+\frac{\partial^{2} T_{i}}{\partial z^{2}}=\frac{1}{a_{i}} \frac{\partial T_{i}}{\partial t}
$$

where the temperature increase $T_{i}$ above the initial temperature of the $i$-layer is a function of the three space variables $(x, y, z)$ and time variable $t$.

The initial temperature field is spatially uniform and the $x$ and $y$-ends boundary conditions are lateral adiabatic walls,

$$
\left.T_{i}\right|_{t=0}=0,\left.\frac{\partial T_{i}}{\partial x}\right|_{x=0 \text { and } L}=0,\left.\frac{\partial T_{1}}{\partial y}\right|_{y=0}=0 \text { and }\left.\frac{\partial T_{2}}{\partial y}\right|_{y=c_{1}+\iota_{2}}=0
$$

$x y$-planes uniform heat losses are characterised by a single convective heat coefficient $h_{c}$ on the upper $(z=e)$ and lower $(z=0)$ faces and the fin hypothesis along $z$-direction is assumed:

$$
\mathbf{n}_{\mathrm{ext}} \cdot \lambda_{i} \nabla T_{i}=h_{c} T_{i} \text { and } \frac{h_{c} e}{\lambda_{i}} \ll 1
$$

At the interface $A_{12}\left(y=e_{1}\right)$ of the phases, the perfect $\left(R_{c}=0\right)$ or resistive contact leads to:

$$
\left.\begin{array}{c}
\mathbf{n}_{12} \cdot \lambda_{1} \nabla T_{1}=\mathbf{n}_{12} \cdot \lambda_{2} \nabla T_{2} \text { and } \\
T_{1}=T \\
\text { or } \\
\frac{1}{R_{c}}\left(T_{1}-T_{2}\right)=-\mathbf{n}_{12} \cdot \lambda_{1} \nabla T_{1}
\end{array}\right\} \text { at } A_{12}
$$

This equation system is solved by suitable integral transform methods for the steady or the transient heat diffusion processes to define a common analytical relationship between averaged temperatures of both phases and the macroscopic thermal impedance $Z$ :

$$
\frac{\left\langle\theta_{1}\right\rangle^{1}}{\left\langle\theta_{2}\right\rangle^{2}}=1+\lambda_{2}^{\prime} \beta_{2}{ }^{2} e_{2} Z \text { with } Z=\sum_{i=1}^{2}\left(\frac{1}{\lambda_{i} \beta_{i} t h\left(\beta_{i} e_{i}\right)}-\frac{1}{\lambda_{i} \beta_{i}{ }^{2} e_{i}}\right)+R_{c}
$$

where in case of steady state $\beta_{2}$ is the generalised frequency of phase 2 :

$$
\beta_{2}^{2}=\alpha^{2}+\frac{2 h_{c}}{\lambda_{2} e}
$$

with the discrete Fourier frequency $\alpha_{j}$. And in case of the transient state:

$$
\beta_{2}^{2}=\frac{p}{a_{2}}+\frac{2 h_{c}}{\lambda_{2} e}
$$

with the Laplace variable $p$.

We introduce the first order term of the Taylor series of the impedance $Z$ for the case that both frequencies $\beta_{1}$ and $\beta_{2}$ are small and we obtain an analytical relationship between the temperature transform ratio, $\lambda_{1}, \lambda_{2}$ and $R_{c}$ : 


$$
\frac{\left\langle\theta_{1}\right\rangle^{1}}{\left\langle\theta_{2}\right\rangle^{2}} \approx 1+\lambda_{2} \beta_{2}{ }^{2} e_{2}\left(\frac{e_{1}}{3 \lambda_{1}}+\frac{e_{2}}{3 \lambda_{2}}+R_{c}\right)=1+\lambda_{2} \dot{\beta}_{2}{ }^{2} e_{2}\left(R+R_{c}\right)
$$

with the intrinsic macroscopic resistance $R$ of the bimodal system.

Therefore, experimental determination of the global resistance $\left(R+R_{c}\right)$ is feasible, provided that experimental temperature data are exploitable with the suited identification method in the frequency space. However, more terms in the Taylor expansion need to be considered for a reliable estimation of the parameters [2] because of the reduced validity frequency range of equation (9). In the next sections, the experimental validations of two methods will be presented for both transient or steady heat conduction processes on samples without thermal interfacial resistance $\left(R_{c}=0\right)$.

\section{Experimental setup}

Steady heat transfer measurements are performed on a stainless steel sample $(3 \mathrm{~mm}$ thick, $42 \mathrm{~mm}$ wide, $655 \mathrm{~mm}$ long). Transient experiments were performed on steel ( $1 \mathrm{~mm}$ thick, $58 \mathrm{~mm}$ wide, $1000 \mathrm{~mm}$ long). Each sample was heated for half of its width, to stand for the two phases. The black painted front face of the slab is scanned by an infrared camera (Avio 2002 ST) at a sampling rate between 4 and 25 frames per second. The infrared radiometer has an array of ten Indium Antimonide sensors coupled to a Stirling cooler. The temperature values of each frame $(256 \times 100)$ are stored in a real-time memory, so that 512 frames of the steady or transient temperature field can be successively recorded.

It is assumed that emissivity of the black coated surface is uniform to avoid systematic measurement errors. The heat source is a flat electric heating element that is glued on the rear face of the 1-phase. Experiments were performed at constant room temperature but room temperature varied from one experiment to the other. Physical locations of the sample are established through visualisation of low emissivity markers on the recorded frames. Both temporal and spatial averaging treatments of the thermal field are performed by the use of the computing environment Matlab [3].

A statistical noise characterisation was carried out for a large number of experimental temperature measurements. Errors of measured temperatures by infrared thermography are well known to be characterised by a zero mean and a constant standard deviation. However, one needs to verify the statistical independence. Several experimental determinations of the covariance matrix of the averaged temperature error vector are performed for a set of $N(=$ 512) recorded infrared frames in the uniform temperature field case. Calculations of the covariance matrix confirm the independence characteristic of the errors. Indeed, covariance values are negligible compared to variance ones.

\section{Steady state inverse problem}

The approximate transformation solution is obtained in the Fourier frequency space and the following identification function can be deduced:

$$
\frac{\left\langle\theta_{1}\right\rangle^{1}}{\left\langle\theta_{2}\right\rangle^{2}} \approx \gamma_{1}+\gamma_{2} \alpha_{j}^{2}+\gamma_{3} \alpha_{j}^{4}+\ldots+\gamma_{m} \alpha_{j}^{2 m-2}+\ldots+\gamma_{k_{y}} \alpha_{j}^{2 N_{y}-2}
$$

where $\gamma_{m}$ are identifiable parameters of the linear model $\left(N_{\gamma}=10\right)$ and $\left(\left\langle\theta_{1}\right\rangle^{1}\right.$, while $\left\langle\theta_{2}\right\rangle^{2}$ ) are intrinsic integral cross-sections (over $y$ variable) averaged temperatures.

Therefore, estimation of $R$ may be processed in the Fourier space for a given number of discrete $\alpha_{j}$ values with infrared temperature fields. These experimental noise data are characterised by additional independent errors with a quantifiable diagonal co-variance matrix (see previous section). The maximum likelihood estimator [4] with minimal variance to identify the parameters $\gamma_{m}$ can be applied. 
In Figs. 2 and 3, an experimental temperature field and its associated macroscopic temperature distributions have been obtained with 512 infrared frames averaged over time to reduce the temperature noise. In Fig. 4, a good agreement between experimental results of $R$ estimations (versus $\alpha_{j}$ ) and simulated ones shows that the method is appropriate to thermal resistance evaluation with infrared measurements for the low frequency range since the signal over noise ratio tends towards unity for higher frequencies.

\section{Transient inverse problem}

The identification function depending on the Laplace variable $\mathrm{p}\left(N_{\gamma}=6\right)$ is

$$
\frac{\left\langle\theta_{1}\right\rangle^{1}}{\left\langle\theta_{2}\right\rangle^{2}} \approx \gamma_{1}+\gamma_{2} p^{1}+\gamma_{3} p^{2}+\ldots+\gamma_{m} p^{m-1}+\ldots+\gamma_{N_{\gamma}} p^{N_{y}-1}
$$

In the case of transient heat conduction, $\left(\left\langle\theta_{1}\right\rangle^{l},\left\langle\theta_{2}\right\rangle^{2}\right)$ are the intrinsic integrai temperatures averaged over $y$ and $x$ space variables so that experimental transient macroscopic temperature evolutions can be obtained from transient infrared temperature fields.

The classical least squares method is used to compute the first approximate set of estimated parameters in the Laplace domain with the thermal diffusivity:

$$
a_{2}=\frac{e_{2}\left(e_{1}+e_{2}\right)}{3 \gamma_{2}}
$$

An iterative procedure (simplex algorithm [4]) allows precise determination of the thermal diffusivity of the sample. The macroscopic temperature evolutions (see Fig. 5) derived this way $\left\langle T_{2}\right\rangle^{2}$ exp are fitted in the time domain with the theoretical ones $\left\langle T_{2}\right\rangle^{2}$ th which are obtained by the convolution product between the experimental evolution $\left\langle T_{1}\right\rangle^{1}$ exp and the inverse Laplace transform of the theoretical function $\left\langle\theta_{2}\right\rangle^{2} /\left\langle\theta_{1}\right\rangle^{\prime}$.

The estimated diffusivity values of the steel sample are in good agreement with the flash method measurements ( $\alpha=1.810^{-5} \mathrm{~m} / \mathrm{s}^{2}$ with a relative standard deviation below $10 \%$ ).

\section{REFERENCES}

[1] RAMOND (L.), BATSALE (J.C.), GOBBÉ (C.) and QUINTARD (M.). - Measurement of a thermal exchange coefficient in porous media by infrared thermography- Two-temperature model application, Inter. Heat Transfer Conf. 11, 1998, Kyongju, Korea.

[2] PONCET (E.), BEREIZIAT (D.), FALLOT (P.), GRANGEOT (G.) and BATSALE (J.C.). Two-medium treatment of heat transfer in layered media: experimental estimation of the heat exchange coefficient by infrared thermography measurement, submitted to Inter. J. Heat and Mass Transfer.

[3] Matlab Reference Guide, The MathsWorks, Inc., 24 Prime Park Way, Natick, Mass. 01760.

[4] PRESS (W.H.), FLANNERY (B.P.), TEUKOLSKY (S.A.) and VETTERLING (W.T.). Numerical recipes, 1989, Cambridge University Press, London. 
http://dx.doi.org/10.21611/qirt.1998.015

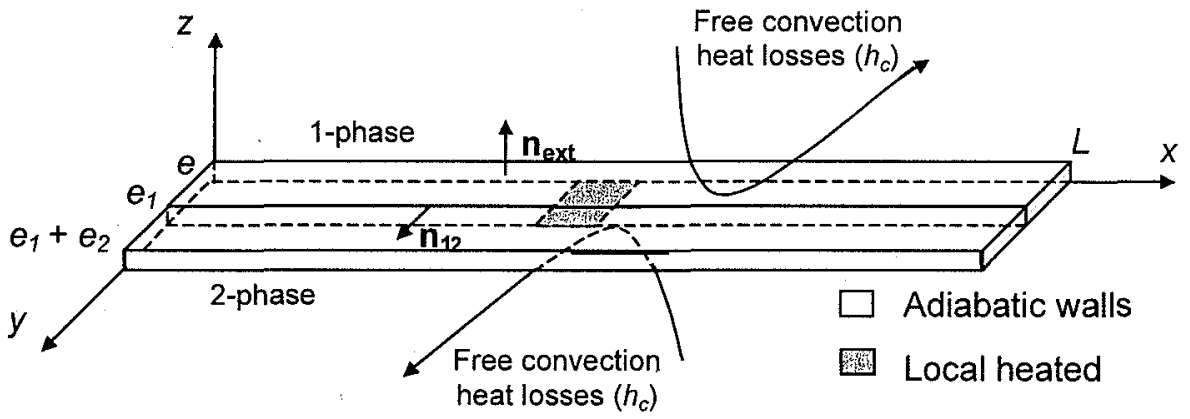

Fig. 1. Geometry and boundary conditions of the system

1-phase

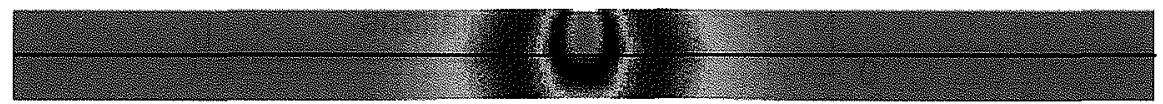

2-phase

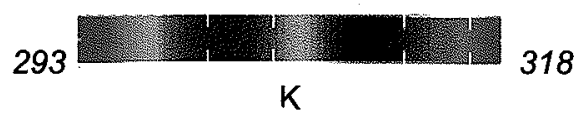

Fig. 2. Steady state thermal experiment: Infrared temperature frame after time averaging process (stainless steel sample)

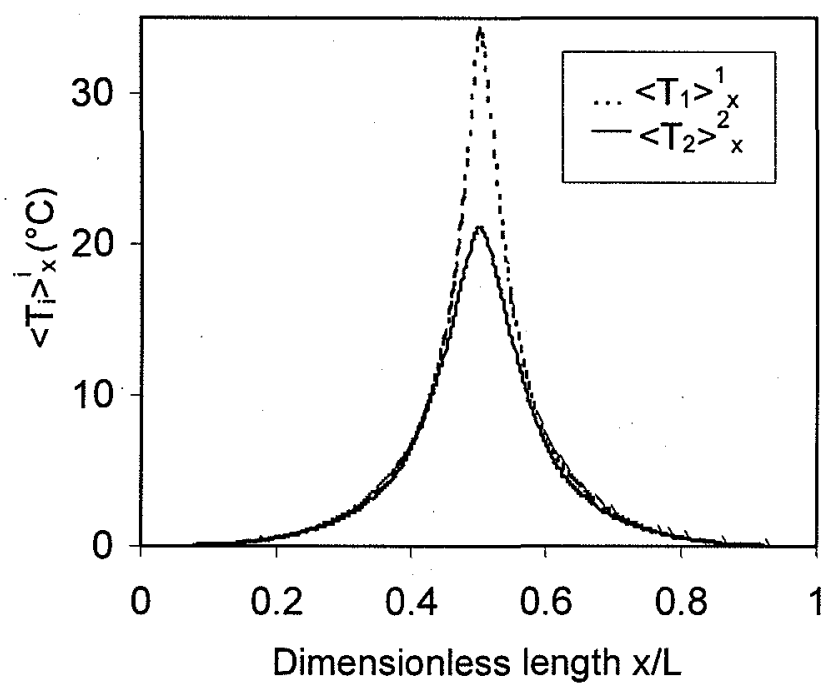

Fig. 3. Steady state thermal experiment: spatial distributions of the cross sectional averaged temperatures (stainless steel sample) 
http://dx.doi.org/10.21611/qirt.1998.015

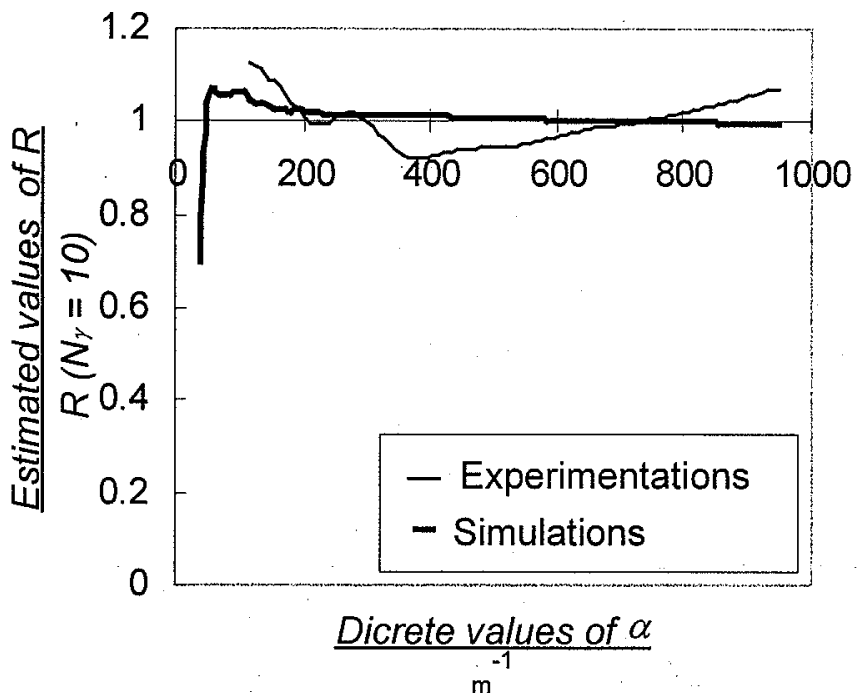

Fig. 4. Steady state experiments: experimental and simulated estimations (stainless steel sample)

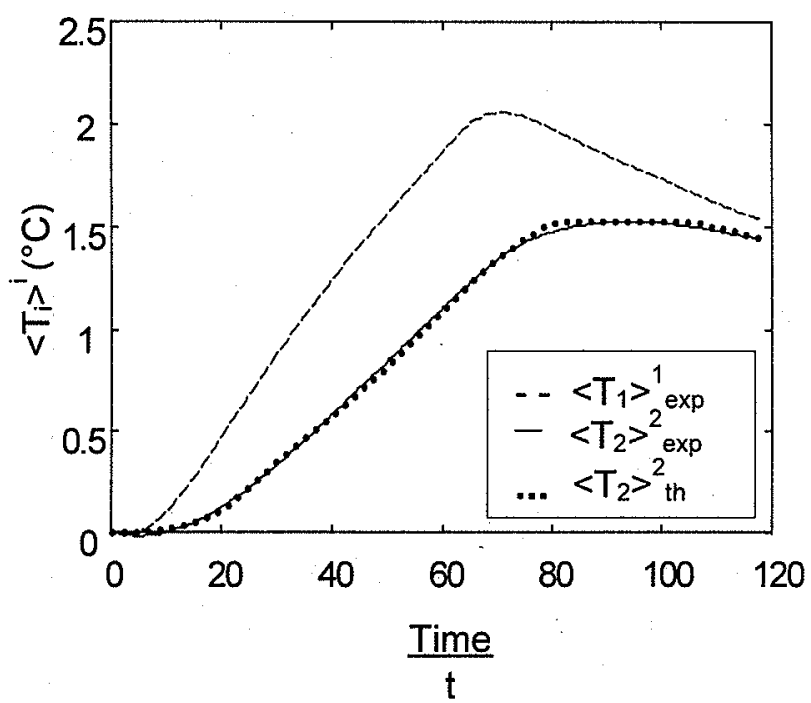

Fig. 5. Transient thermal experiment: evolutions of the surface averaged temperatures (steel sample) 